\title{
Treatment of multiple sclerosis by transplantation of neural stem cells derived from induced pluripotent stem cells
}

\author{
Chao Zhang ${ }^{1,2}$, Jiani $\mathrm{Cao}^{1}$, Xiaoyan $\mathrm{Li}^{1}$, Haoyu Xu ${ }^{1,2}$, Weixu Wang ${ }^{1}$, Libin Wang ${ }^{1,2}$, \\ Xiaoyang Zhao ${ }^{1}$, Wei $\mathrm{Li}^{1}$, Jianwei Jiao ${ }^{1}$, Baoyang $\mathrm{Hu}^{1}$, Qi Zhou ${ }^{1 *} \&$ Tongbiao Zhao ${ }^{1 * *}$ \\ ${ }^{1}$ State Key Laboratory of Stem Cell and Reproductive Biology, Institute of Zoology, Chinese Academy of Sciences, Beijing 100101, China; \\ ${ }^{2}$ Graduate University of Chinese Academy of Sciences, Beijing 100049, China
}

Received April 28, 2016; accepted May 11, 2016; published online May 26, 2016

\begin{abstract}
Multiple sclerosis (MS) is an autoimmune disease of the central nervous system (CNS), with focal T lymphocytic infiltration and damage of myelin and axons. The underlying mechanism of pathogenesis remains unclear and there are currently no effective treatments. The development of neural stem cell (NSC) transplantation provides a promising strategy to treat neurodegenerative disease. However, the limited availability of NSCs prevents their application in neural disease therapy. In this study, we generated NSCs from induced pluripotent stem cells (iPSCs) and transplanted these cells into mice with experimental autoimmune encephalomyelitis (EAE), a model of MS. The results showed that transplantation of iPSC-derived NSCs dramatically reduced $\mathrm{T}$ cell infiltration and ameliorated white matter damage in the treated EAE mice. Correspondingly, the disease symptom score was greatly decreased, and motor ability was dramatically rescued in the iPSC-NSC-treated EAE mice, indicating the effectiveness of using iPSC-NSCs to treat MS. Our study provides pre-clinical evidence to support the feasibility of treating MS by transplantation of iPSC-derived NSCs.
\end{abstract}

induced pluripotent stem cell, multiple sclerosis, neural stem cell, regenerative medicine, transplantation

Citation: Zhang, C., Cao, J., Li, X., Xu, H., Wang, W., Wang, L., Zhao, X., Li, W., Jiao, J., Hu, B., Zhou, Q., and Zhao, T. (2016). Treatment of multiple sclerosis by transplantation of neural stem cells derived from induced pluripotent stem cells. Sci China Life Sci 59, 950-957. doi: $10.1007 / \mathrm{s} 11427-016-0114-9$

\section{INTRODUCTION}

Multiple sclerosis (MS) is a neurodegenerative disease, which leads to axon loss and myelin damage in the central neural system (CNS) of patients (Compston and Coles, 2008). Currently, the exact pathological mechanism of MS is still unclear. One hypothesis suggests that MS is an autoimmune disease. Lymphocytic cells, recruited by the autoimmune response occurring in the CNS, disrupt the blood-brain barrier and infiltrate the CNS. Immune cells that reach the CNS may survive for a long time, inducing demyelination and irreversible axon loss (Arima et al.,

*Corresponding author (email: qzhou@ioz.ac.cn)

***Corresponding author (email: tbzhao@ioz.ac.cn)
2013; Viglietta et al., 2004). Currently, clinical MS therapies mainly rely on immunomodulatory drugs such as natalizumab, mitoxantrone, and azathioprine (Constantinescu et al., 2011; Jelinek et al., 2015; Kleinschmidt-DeMasters and Tyler K, 2005). These drug treatments can alleviate disease symptoms and reduce relapse frequency to some extent. However, drug treatment cannot rescue the neuronal damage within the neural lesions. Remyelination and neural cell regeneration are still major hurdles for MS treatments.

Recent progress and remarkable advancement in stem cell research has led to an exciting prospect of regenerative medicine and provided new strategies to treat neurodegenerative disease (Huang and $\mathrm{Fu}, 2014$ ). Human embryonic stem cell (ESC)-derived NSCs transplanted into the Parkinson's rodent model have been reported to successfully gen- 
erate DA neurons in the host and ameliorate the disease (Ben-Hur et al., 2004; Kim et al., 2002; Redmond et al., 2007). Detailed tracing experiments showed that human NSCs injected into a Huntington's mouse model migrate into the striatal lesion and promote long-term recovery of neural function (Lee et al., 2005). Interestingly, intrahippocampal injection of human NSCs promoted cognitive function in an Alzheimer's mouse model, indicating that transplanted NSCs can functionally integrate into the host (Ager et al., 2015). Furthermore, stem cell transplantation has also provided new strategies to treat neural autoimmune disease. Early reports showed that transplantation of adult neural progenitor cells promoted neural function recovery in the EAE mouse model of multiple sclerosis. A recent study suggested that transplantation of bone marrow derived mesenchymal stem cells (MSCs) alleviated the progression of MS-like symptoms in EAE mice (Harris et al., 2012). These experiments support the development of neural stem cells to treat diseases that involve neural degeneration or damage. However, the limited availability of neural stem cells restricts the large-scale expansion of neural stem cell therapy (Kim et al., 2012; Pluchino and Martino, 2008; Pluchino et al., 2003).

Induced pluripotent stem cells (iPSCs) exhibit transcriptional and epigenetic characteristics that are highly similar to those of ESCs (Takahashi and Yamanaka, 2006). Similar to ESCs, iPSCs can undergo self-renewal and maintain pluripotency to differentiate into any cell type. Thus iPSCs can potentially provide unlimited autologous cells for therapy, and hold great promise for regenerative medicine. Safety and efficacy are the most important questions for clinical development of iPSCs. The therapeutic potential of iPSCs has been extensively assessed by transplantation of iPSC-derived specific cells into different animal disease models (Liu et al., 2014; Lu and Zhao, 2013). However, at the same time, it has been reported that the residual undifferentiated cells inside the iPSC-differentiated specific lineage might cause tumor formation after transplantation (Liu et al., 2014; Lu and Zhao, 2013). Furthermore, it has been shown that certain cells derived from iPSCs can elicit antigen-specific immune rejection responses after transplantation into the autologous recipients (Zhao et al., 2011, 2015). Thus it is necessary to extensively examine the safety and efficacy of iPSC-NSCs for therapy.

Mice with experimental autoimmune encephalomyelitis (EAE) are widely used to model MS. EAE has many similarities to MS, such as demyelination and axon loss (Constantinescu et al., 2011). In this study, we differentiated C57BL/6 mouse iPSCs into NSCs in vitro and transplanted these cells into EAE mice to evaluate their therapeutic effects. We found that transplanted NSCs derived from iPSCs in vitro were integrated into the nervous system and the infiltration of $\mathrm{T}$ cells into the CNS was greatly reduced. Correspondingly, NSC transplantation promoted remyelination and improved the recovery of motor function in the EAE mice.

\section{RESULTS}

\section{Induction of the EAE model}

To investigate the therapeutic efficacy of iPSC-NSC transplantation, we first established EAE in mice as a model of MS. EAE was induced by injecting mice with myelin oligodendrocyte glycoprotein peptide 35-55 ( $\left.\mathrm{MOG}_{35-55}\right)$ emulsified in complete Freund's adjuvant (CFA). The level of disease symptoms was assessed with a standard score ranging from 0 to 5 (Figure 1A). Mice that scored 3 (severe hindlimb weakness) were sacrificed and the spinal cords were stained with hematoxylin-eosin (HE) (Figure 1A). Compared to normal control mice, the EAE mice had serious tissue necrosis and dispersed cell infiltration at the edge of the white matter, suggesting that the CNS was destroyed in the diseased mice (Figure 1B). Anti-MBP staining showed marked demyelination at the outer edge of the white matter in EAE mice but not normal controls (Figure 1B and C). Furthermore, staining the sections with anti-CD3, anti-CD4 and anti-CD8 antibodies revealed extensive infiltration of $\mathrm{T}$ cells into the spinal cords of the EAE mice but not the normal controls (Figure 1D).

The Treadscan system was used to collect, analyze, and quantify gait data from the EAE mice and normal mice based on a video recording of mice walking in a relaxed state. The disability of EAE mice was characterized by four different indexes: swing time of rear feet, stride time of rear feet, stride length and average instant running speed. We found that the swing time and stride time of the rear feet were much longer in EAE mice than normal mice. The stride length and average instant running speed of rear foot were lower than those of normal mice (Figure 1E). Furthermore, open-field assays showed that the total running distance, the time spent in the central area and the number of times the animals entered the central area was lower for EAE mice than for normal mice, further confirming the limited mobility of EAE mice (Figure 1F).

\section{Differentiation of NSCs from iPSCs in vitro}

We used a modified monolayer neural differentiation protocol to differentiate iPSCs into NSCs (Figure 2A) (Okabe et al., 1996; Ying et al., 2003). To get more pure neural stem cells for transplantation, the cells were dissociated by trypsin on day 12 of differentiation and plated into a new plate for continue culturing. The differentiated cells were aggregated and propagated as neural spheres (Figure $2 \mathrm{~A}$ and B). Further characterization showed that these cells were positive for Nestin and Sox2, like primary neural stem cells. Most importantly, these iPSC-derived cells can differentiate into Tuj1-positive neurons, indicating their neural differentiation potential (Figure 2D).

\section{Transplantation of iPSC-NSCs effectively improves the functional recovery of EAE mice}

To investigate whether the transplanted NSCs can integrate into the host and functionally repair the damaged neural 


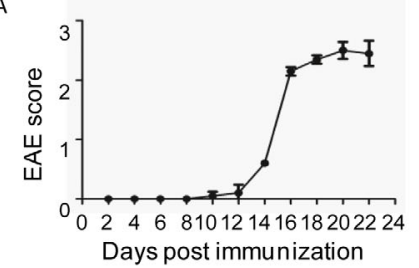

B

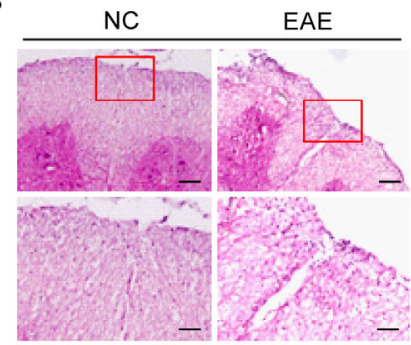

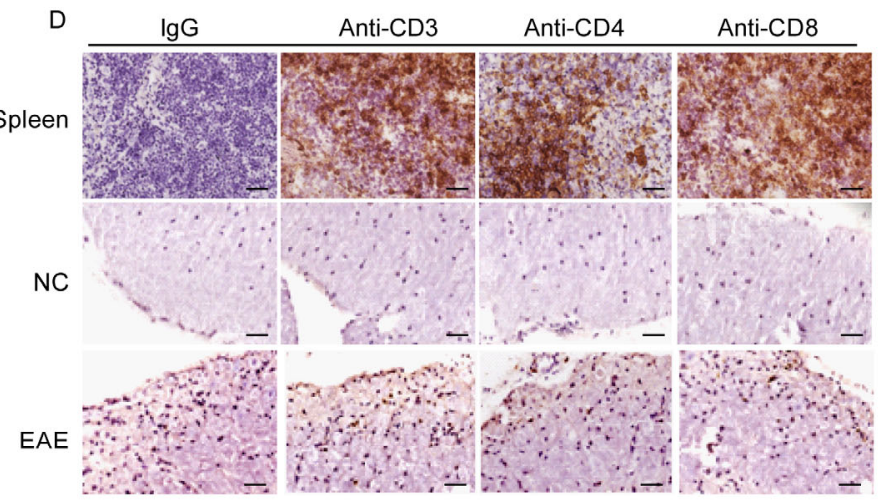

E

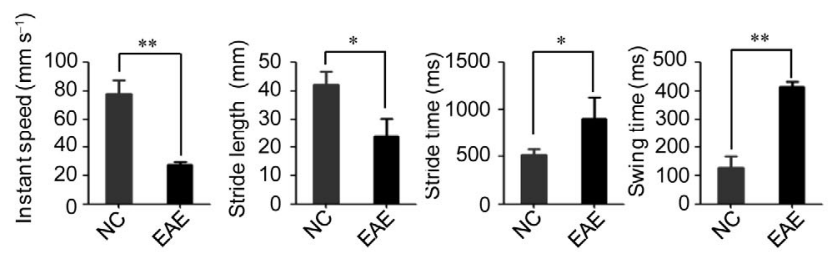

C

MBP

DAPI

Merge

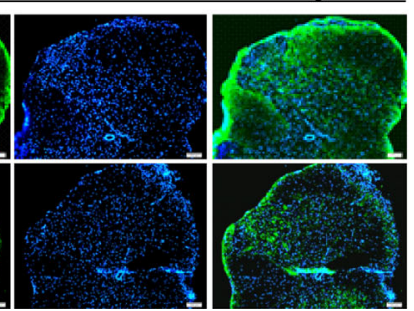

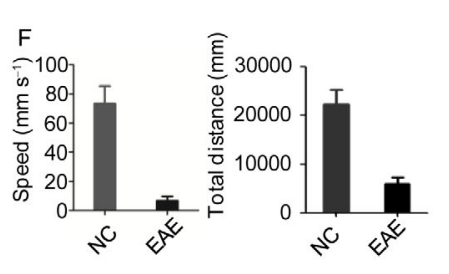

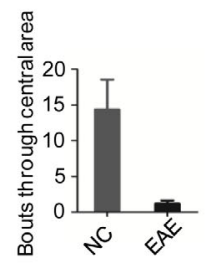

Figure 1 Characterization of EAE mice. A, Mice immunized with $\mathrm{MOG}_{35-55}$ show an increased score of disease symptoms. B, Images of H\&E-stained sections of spinal cord from normal and EAE mice. Scale bars, $200 \mu \mathrm{m}$ (Top), $100 \mu \mathrm{m}$ (Bottom). C, EAE mice show demyelination and axon loss. Spinal cord sections from EAE and normal mice were stained with anti-MBP antibody and DAPI, and analyzed by fluorescence microscopy. Blue, DAPI. Green, MBP. Scale bars, $200 \mu \mathrm{m}$. D, The spinal cords of EAE mice are extensively infiltrated with T cells. T cells were identified with anti-CD3, anti-CD4 and anti-CD8 antibodies. Sections from the spleen were used as a positive control. Scale bars, $100 \mu$ m. E, EAE mice show compromised mobility. The instant running speed, stride lengths, stride time and swing time of EAE mice were determined by GaitScan. Data shown as mean \pm standard deviation (SD), $n=3$; $*, P<0.05$. **, $P<0.01$, Student's $t$-test. F, EAE mice have defective spontaneous movement compared to normal mice. An open-field experiment was used to determine the average speed, the total running distance and the number of entries into the central area.

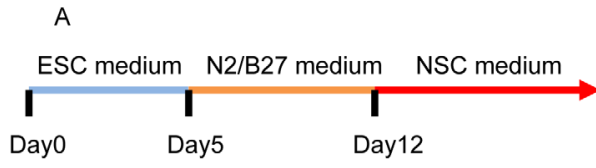

C

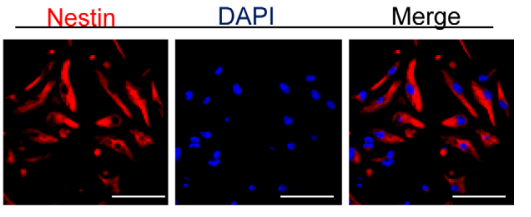

B
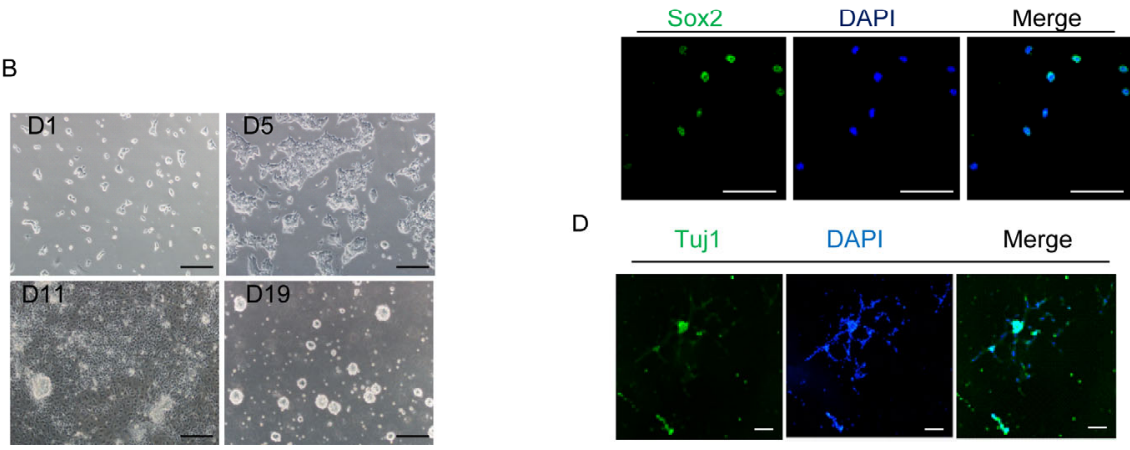

Figure 2 Generation and characterization of neural stem cells derived from B6 iPSCs. A, Scheme of neural stem cell differentiation from iPSCs in vitro. B, Morphology of neural progenitor cells at distinct stages of differentiation. Scale bars, $200 \mu \mathrm{m}$. C, Neural stem cells derived from B6 iPSCs (iPSC-NSCs) express Nestin and Sox2. Red, Nestin. Green, Sox2. Blue, DAPI. Scale bars, $50 \mu \mathrm{m}$. D, iPSC-NSCs can differentiate into Tuj1-positive neurons. Green, Tuj1. Blue, DAPI. Scale bars, $100 \mu \mathrm{m}$. 
tissues, the iPSC-NSCs were labeled with a lentivirus vector expressing mCherry (pCDH-mCherry) to trace their location after transplantation (Figure 3A). The mCherry-labeled iPSC-NSCs were transplanted into the lateral ventricle of EAE mice. The brains of the EAE mice were extensively examined at different days after administration of iPSCNSCs, and mCherry-RFP-positive cells were routinely detected in the dentate gyrus within one month of transplantation (Figure 3B). Most importantly, mCherry-RFP was detected to co-localize with the neuron marker Tju1 in the lesion areas, indicating the integration and differentiation of iPSC-NSC-derived neurons into the damaged tissues (Figure $3 \mathrm{~B}$ ).

The structural and functional recovery of the EAE mice was monitored for two months after iPSC-NSC injection. Using anti-MBP antibody staining, we found that transplantation of iPSC-NSCs but not allogeneic NSCs leads to remyelination in the marginal zone of the white matter (Figure 3C). Correspondingly, iPSC-NSCs but not allogeneic NSCs greatly decreased the EAE score, indicating the functional recovery of the EAE mice by iPSC-NSC treatment (Figure 3D). Furthermore, we performed Treadscan analysis to investigate motor function recovery, and found that transplantation of iPSC-NSCs but not allogeneic NSCs dramatically improved the motor ability of EAE mice (Figure $3 \mathrm{E}$ and $\mathrm{F}$ ). Together, these data suggest that transplantation of iPSC-NSCs can effectively promote remyelination of the damaged spinal cords, alleviate EAE disease symptoms, and improve motor function.

\section{Immunogenicity analysis of iPSC-NSCs}

It has been shown that certain cell types derived from iPSCs, like smooth muscle cells and endothelium cells, can be rejected after transplantation into autologous recipients

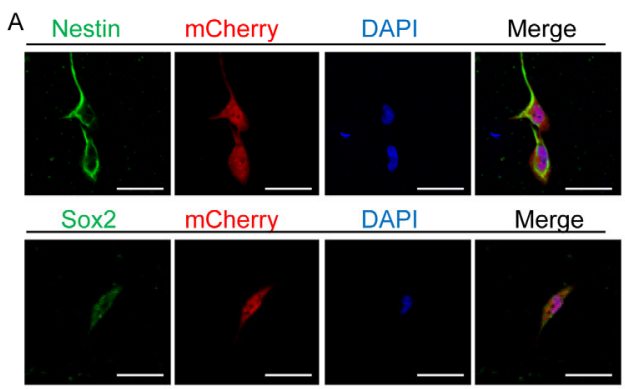

B

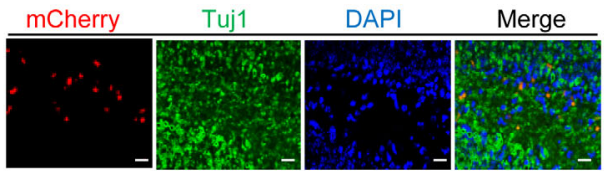

D

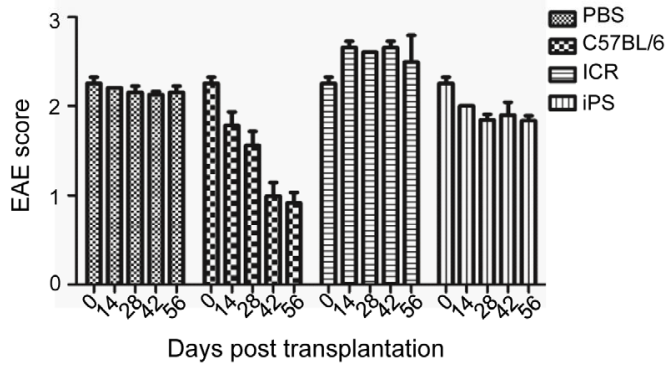

C
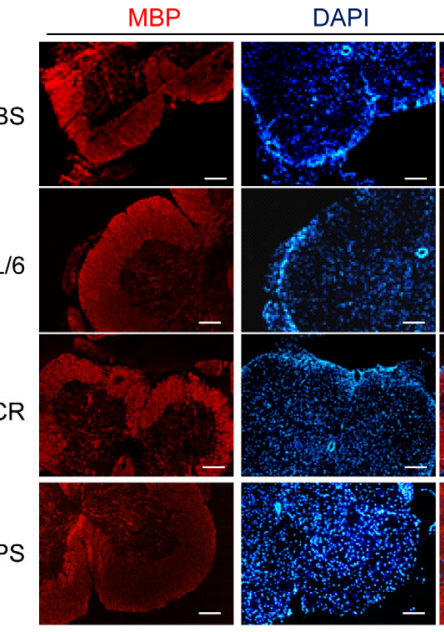

Merge

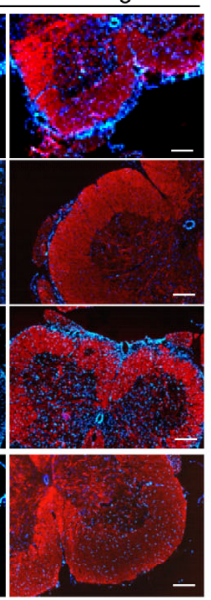

E

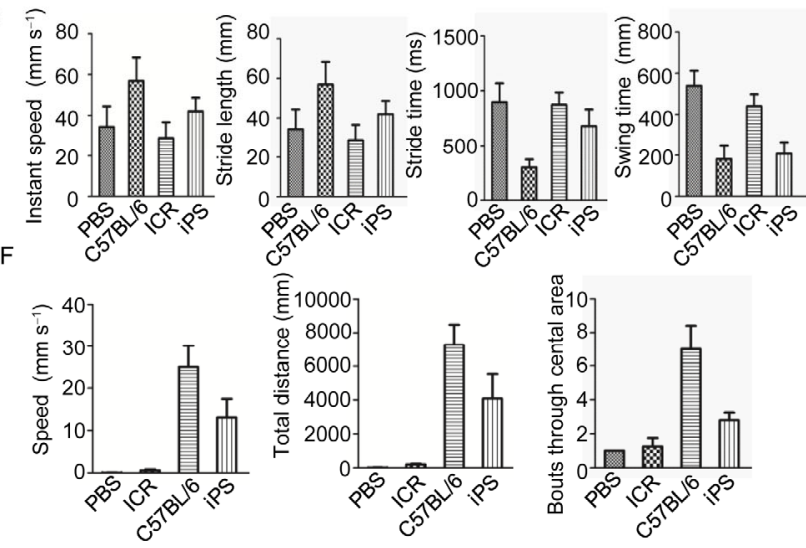

Figure 3 Transplantation of iPSC-NSCs improves the motor function of EAE mice. A, The iPSC-NSCs are marked with pCDH-mCherry for cell fate tracing after transplantation. Green, Nestin and Sox2. Red, mCherry. Blue, DAPI. Scale bars, $50 \mu \mathrm{m}$. B, The transplanted iPSC-NSCs differentiate into neurons and integrate into the lesions in EAE mice. Red, mCherry. Green, Tju1. Blue, DAPI. Scale bars, $100 \mu \mathrm{m}$. C, EAE mice with transplanted iPSC-NSCs show remyelination in the lesion area. Red, MBP. Blue, DAPI. Scale bars, $200 \mu \mathrm{m}$. D, EAE symptoms are improved by iPSC-NSC transplantation. NSCs isolated from syngeneic C57BL/6 mice and allogeneic ICR mice were transplanted into the EAE mice, and used as positive and negative controls respectively. E, The mobility of EAE mice is recovered by iPSC-NSC transplantation. Stride length, instant speed, stride time and swing time were determined by GaitScan analysis. NSCs isolated from syngeneic C57BL/6 mice and allogeneic ICR mice were transplanted into EAE mice, and used as positive and negative controls respectively. F, Open-field experiment showing recovery of mobility in EAE mice treated with iPSC-NSC transplantation. Entries into the central area, total distance and average speed were recorded and analyzed by GaitScan software. NSCs isolated from syngeneic C57BL/6 mice and allogeneic ICR mice were transplanted into EAE mice, and used as positive and negative controls respectively. 
(Araki et al., 2013; de Almeida et al., 2014). To investigate whether NSCs derived from iPSCs can elicit immune rejection responses and thus affect their clinical applications, $\mathrm{T}$ cell stimulation assays were performed. Splenic $\mathrm{T}$ cells were isolated from B6 mice that received NSC transplantation, labeled with carboxy fluorescein succinimidyl ester (CFSE), co-cultured with syngeneic iPSC-NSCs or allogeneic NSCs, and then analyzed with flow cytometry. T cells co-cultured with NSCs proliferated extensively, while T cells stimulated with iPSC-NSCs were not induced to proliferate, indicating that iPSC-NSCs have limited immunogenicity (Figure 4A and B).

To investigate whether iPSC-NSC transplantation alleviates the autoimmune disease characteristics of EAE, we performed immune staining of spinal cords from EAE mice treated with ICR-NSCs or iPSC-NSCs. Transplantation of iPSC-NSCs, but not ICR-NSCs, greatly reduced the infiltration of $\mathrm{T}$ cells into the spinal cords of EAE mice (Figure 4C). This indicates that iPSC-NSC transplantation not only repaired the damaged neurons but also alleviated the autoimmune rejection responses.

\section{DISCUSSION}

Recent reports showed that both MSC and hematopoietic stem cells (HSC) transplantation had impressive therapeutic effects on MS when compared to traditional drug treatments (Fisher-Shoval et al., 2012; Lajimi et al., 2012). Mechanistic studies indicated that the transplanted human MSCs sta- bilized the symptoms in progressive MS and prevented severe exacerbation, whereas the HSCs played a role in immune modulation, which could reduce relapse, relieve symptoms and slow the progression of disability after transplantation (Fassas et al., 1997). However, transplantation of HSCs and/or MSCs could not repair damaged neural cells and myelin, and thus could stabilize the progression of disease but could not cure it. NSCs can be maintained in vitro and differentiated into distinct types of neural cells, making them an ideal resource for therapy. A number of studies have demonstrated that neural progenitor cell transplantation significantly promotes the recovery of damaged neurons in both mouse models (Bai et al., 2013; Einstein et al., 2003; Kim et al., 2012; Wu et al., 2013) and primate models (Pluchino et al., 2009). However, a lack of neural progenitor sources and immune rejection are the two bottlenecks restricting the development of this new MS treatment strategy. NSCs derived from patients' own iPSCs can potentially resolve these issues (Cundiff and Anderson, 2011; Durnaoglu et al., 2011; Israel et al., 2012). In this study, we generated mouse iPSCs, differentiated these cells into NSCs in vitro, transplanted them into EAE mice, and evaluated their therapeutic efficacy. The transplanted NSCs can differentiate into neurons and integrate into damaged tissues in the recipient. Correspondingly, the iPSC-NSC treatment greatly alleviated the disease phenotype and improved the mobility of EAE mice.

MS is an autoimmune disease in which the underlying pathological mechanism remains unclear. Transplantation of
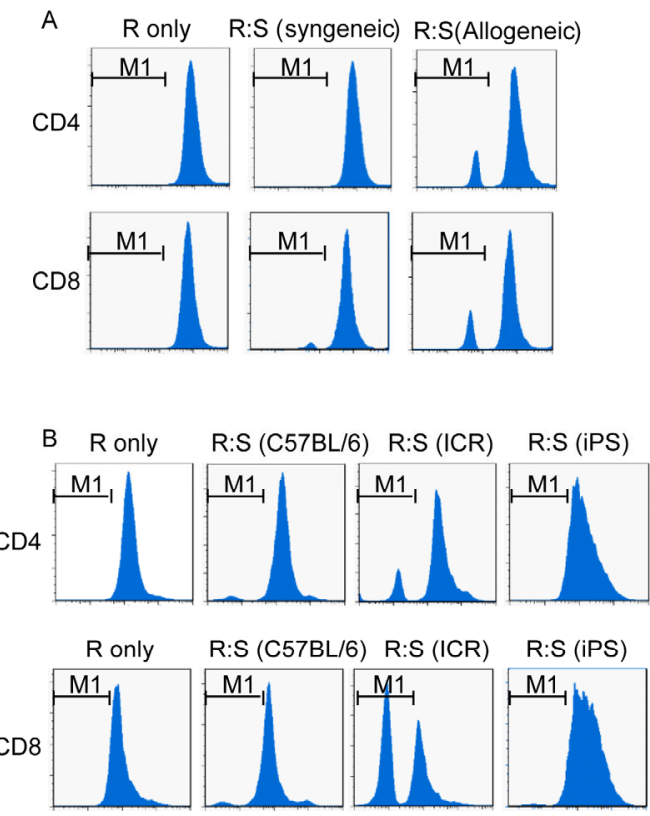

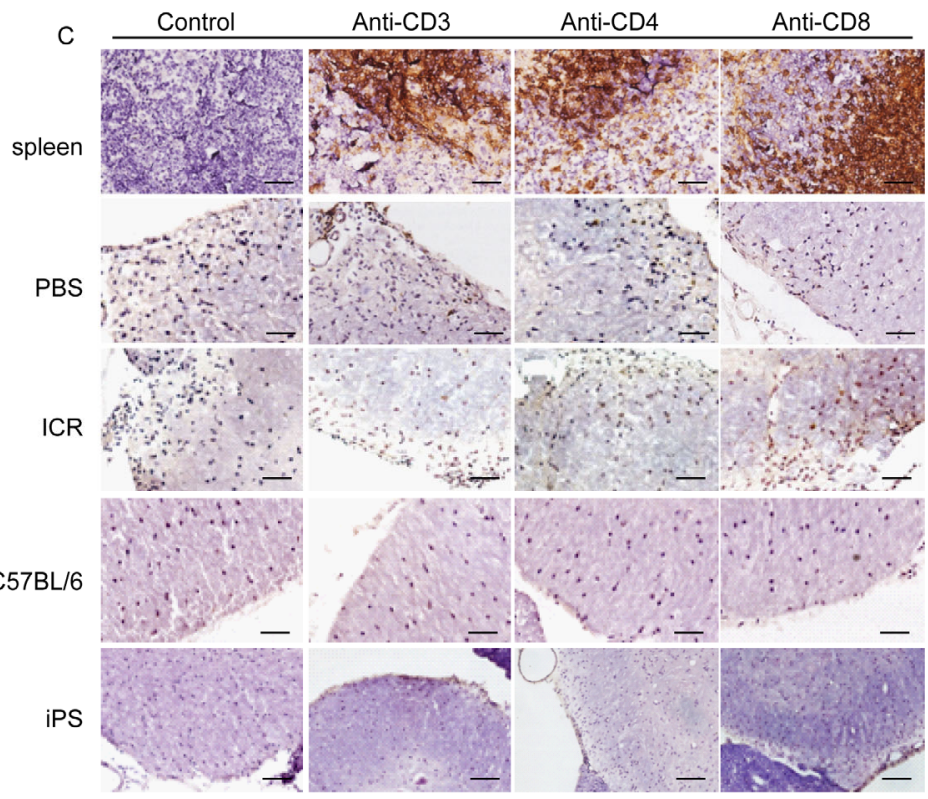

Figure 4 iPSC-NSC transplantation reduces T cell infiltration. A, T cells are activated by allogeneic but not syngeneic NSCs. CD4 ${ }^{+}$and CD $8^{+}$T cells were stained with CFSE and co-cultured with allogeneic or syngeneic NSCs. The proliferation of T cells was determined by a flow cytometry. B, In vitro differentiated iPSC-NSCs do not induce an immune rejection response. CD4 ${ }^{+}$and $\mathrm{CD}^{+} \mathrm{T}$ cells were isolated from $\mathrm{C} 57 \mathrm{BL} / 6$ mice receiving PBS, allogeneic ICR NSCs, syngeneic C57BL/6 NSCs or iPSC-NSCs, and were co-cultured with the grafts. C, Transplantation of iPSC-NSCs reduces infiltration of T cells inside the spinal cord of EAE mice. T cells were identified by anti-CD3, anti-CD4 and anti-CD8 antibodies. Sections from the spleen were used as a positive control. Scale bars, $100 \mu \mathrm{m}$. 
HSCs/MSCs cannot repair damaged neurons, but dramatically alleviates the immune attack, preventing disease deterioration. Surprisingly, the iPSC-NSC transplantation in our study not only repaired the damaged tissues, but also dramatically decreased $\mathrm{T}$ cell infiltration in the treated EAE mice. The mechanisms underlying the reduction of inflammation in the CNS are unclear. One possibility is that the tissues repaired by iPSC-NSC transplantation lose the ability to further recruit new lymphocytic cells.

In 2011, we used a teratoma formation assay to show that mouse iPSC derivatives can be immunogenic. However, we did not identify at that time which kinds of cell/tissue inside the teratoma elicited the immune rejection response (Zhao et al., 2011). One follow-up study has shown that cardiomyocytes but not skin cells derived from mouse iPSCs can induce $\mathrm{T}$ cell infiltration after transplantation (Araki et al., 2013). In support of that view, a recent study has shown that autologous endothelial cells derived from iPSCs can elicit an immune response that resembles the one against self. Interestingly, these iPSC-derived autologous endothelial cells prompt a tolerance response characterized by increased expression of IL-10 (de Almeida et al., 2014). In this study, we did not observe an immune rejection response upon iPSC-NSC transplantation. Together, these studies suggest that certain cells derived from iPSCs are immunogenic, while others are not (Cao et al., 2014; Zhao et al., 2015).

The successful repair of damaged neural cells and myelin, alleviation of $\mathrm{T}$ cell infiltration, and improvement of motor ability upon iPSC-NSC administration in EAE mice indicate that iPSC-NSCs can integrate into the recipient and cure the disease. These studies support the feasibility of treating MS using autologous NSCs derived from iPSCs. Further experiments using human or non-human primate iPSC-derived NSCs to evaluate the therapeutic efficacy will significantly speed up the clinical development of iPSCderived NSCs.

\section{MATERIALS AND METHODS}

\section{Induction of EAE and administration of NSCs}

All mice used were purchased from Vital River Inc China. Seven-week-old male C57BL/6 mice with an average body weight ranging from 17-19 g were selected and immunized subcutaneously with emulsion contained $200 \mu \mathrm{g} \mathrm{MOG}_{35-55}$. The $\mathrm{MOG}_{35-55}$ was diluted in $100 \mu \mathrm{L}$ phosphate buffer saline (PBS), $100 \mu \mathrm{L}$ complete Freund's adjuvant (CFA, Sigma, USA), and $10 \mathrm{mg} \mathrm{mL}^{-1}$ Mycobacterium tuberculosis (H37Ra) (TB, Difco, USA). The mice were then injected with $1 \mu \mathrm{g} \mu \mathrm{L}^{-1}$ pertussis toxin (PT, Sigma) twice within $48 \mathrm{~h}$. All mice were monitored daily for clinical signs of EAE and evaluated by a standard disease score ranging from 0 to $5: 0$, no abnormality. 1, limp tail. 2, mild hindlimb weakness. 3, severe hindlimb weakness. 4, complete hindlimb paralysis. 5, death. When the mice showed severe hindlimb weakness (usually 18 days after disease induction), they were divided into four groups and treated with PBS, syngeneic C57BL/6 NSCs, allogeneic ICR NSCs and iPSC-NSCs. Each group contains 10 mice for transplantation. All NSCs were delivered into mice by intraventricular injection. Briefly, mice were anesthetized and fixed on the platform of the stereotaxic apparatus. Each mouse brain was injected with $2 \times 10^{5}$ cells in $10 \mu \mathrm{L}$ PBS. Disease symptoms were usually monitored for three months.

\section{In vitro differentiation from iPS cells to neural stem cells}

Neural stem cell differentiation from iPSCs was performed by modifying a published protocol (Okabe et al., 1996; Ying et al., 2003). Briefly, undifferentiated iPS cells were seeded on a $0.1 \%$ gelatin-coated plate with a density of $1 \times 10^{4} \mathrm{~cm}^{-2}$ in ES medium without LIF for five days. At day 6, ES culture medium (knockout Dulbecco's modified eagle's medium with $15 \%$ fetal bovine serum, $2 \mathrm{mmol} \mathrm{L}^{-1}$ glutamine, $1 \mathrm{mmol} \mathrm{L}^{-1}$ sodium pyruvate, $0.1 \mathrm{mmol} \mathrm{L}^{-1}$ nonessential amino acids, $100 \mu \mathrm{g} \mathrm{mL}^{-1}$ streptomycin, $100 \mathrm{U} \mathrm{mL}^{-1}$ penicillin, $0.055 \mathrm{mmol} \mathrm{L}^{-1} \beta$-mercaptoethanol, and 1,000 $\mathrm{U} \mathrm{mL}^{-1}$ leukemia inhibitory factor) was changed to a modified NSC differentiation N2B27 medium. The N2B27 medium is a 1:1 mixture of DMEM/F12 (Gibco, USA) supplemented with modified $\mathrm{N} 2\left(25 \mu \mathrm{g} \mathrm{mL}{ }^{-1}\right.$ insulin, $100 \mu \mathrm{g} \mathrm{mL}^{-1}$ transferrin, $6 \mathrm{ng} \mathrm{mL} \mathrm{m}^{-1}$ progesterone, $16 \mu \mathrm{g} \mathrm{mL} \mathrm{m}^{-1}$ putrescine, $30 \mathrm{nmol} \mathrm{L}{ }^{-1}$ sodium selenite and $50 \mu \mathrm{g} \mathrm{mL}^{-1}$ bovine serum albumin fraction $\mathrm{V}$ ) (Gibco) and Neuron basal medium supplemented with B27 (both from Gibco). The NSC differentiation medium was replaced every two days. After seven days culturing, cells were dissociated with $0.05 \%$ trypsin/0.04\% EDTA and transferred to a non-adherent plate. These cells were then cultured in NSC medium which was Neuron basal medium supplemented with $\mathrm{N}-2$ supplement $(1: 100)$, B-27 supplement $(1: 50), 10 \mu \mathrm{g} \mathrm{mL}{ }^{-1}$ bFGF and EGF.

\section{$T$ cell activation assay}

$\mathrm{CD}^{+}$and $\mathrm{CD}^{+} \mathrm{T}$ cells were isolated from the mouse spleens. Spleens were pulverized and fractionated on a Percoll gradient. The isolated cells were washed twice with $\mathrm{PBS}$, then $\mathrm{CD}^{+}$and $\mathrm{CD}^{+} \mathrm{T}$ cells were sorted using a magnetic purification kit (Miltenyi, Germany). The $\mathrm{CD}^{+}$ and $\mathrm{CD}^{+}$cells (responder, R) were labeled with CFSE (Invitrogen, USA) and co-cultured with the corresponding NSCs (stimulator, S) for $72 \mathrm{~h}$. Flow cytometric analysis was performed to determine the activation of T cells.

\section{Immunohistochemistry}

The tissue samples were fixed in $4 \%$ paraformalin overnight at $4^{\circ} \mathrm{C}$ and immersed in $30 \%$ sucrose solution overnight for dehydration. Tissues were then embedded in OCT for sectioning $(10 \mu \mathrm{m})$. The sections were stained by anti-CD3, 
anti-CD4 and anti-CD8 antibodies (eBioscience, USA). Staining with primary antibodies was performed with an immunohistochemistry kit (ZSGB-BIO, Beijing). All images were captured using a Leica SCN400 scanner (Leica, Japan). For cell samples, cells cultured on cover slips were fixed in $4 \%$ paraformalin for 10 minutes, and rinsed in $0.1 \%$ Triton for $1 \mathrm{~h}$. Then slips were blocked by $0.2 \% \mathrm{BSA}$ for $1 \mathrm{~h}$ and incubated with primary antibodies against Nestin (1:300, Millipore, USA), Sox2 (1:500, Abcam, UK), or MBP (1:400, Santa Cruz, USA) overnight at $4^{\circ} \mathrm{C}$. Cells were washed twice with PBS and incubated with AlexaFluorconjugated secondary antibodies (1:2000, Invitrogen) for 1 h. 4',6-diamidino-2-phenylindole (DAPI) was added $30 \mathrm{~min}$ before observation $(1: 500$, Invitrogen). All images were captured using a Zeiss LSM 700 confocal laser-scanning microscope (Zeiss, Germany).

\section{Treadscan analysis}

The Treadscan analyses were designed according to a published protocol (Beare et al., 2009). Briefly, the device consists of a motor-driven transparent belt with a mirror below, a high-speed digital video camera which recorded the view reflected from the belt by the mirror, and a transparent $17 \mathrm{~cm} \times 5 \mathrm{~cm}$ compartment above the belt which ensured that the mouse stayed in place. Treadscan software (CleverSys) recognized each paw image of the mouse in order to analyze the walking pattern. All groups of mice were trained for two weeks from one week before immunization to one week after immunization, and each week contained four sessions. In a training session, mice were trained to walk on the motor-driven belt at a constant speed of $20 \mathrm{~cm} \mathrm{~s}^{-1}$ for $1 \mathrm{~min}$. Because sick (or EAE induced) mice were unable to reach a speed of $20 \mathrm{~cm} \mathrm{~s}^{-1}$, a lower speed of $5 \mathrm{~cm} \mathrm{~s}^{-1}$ was set for them. Mice that received two weeks training were recorded by video two months post immunization. Each mouse had three videos taken and they ran on the belt for 30 s per video. Videos in which the animals ran continuously without stopping were used for analysis. The outlines of paws were deleted to make sure the data reflected the normal walking patterns of the mice. Four indexes-instant speed, swing time, stride time, and stride length—were collected.

\section{Open-field test}

The open-field test is an experiment to evaluate general locomotor activity levels and anxiety in rodents (Crusio, 2001). At the beginning of the test, the arena was cleaned to remove any residual scent from other mice. All groups of mice were tested one month after immunization. Each mouse was placed in the center of the square arena. A high-speed digital camera was fixed on top of the arena to record the movement of the mice in the arena for five min. Videos were analyzed by Topscan software. A central area was defined in the floor of the arena, consisting of a concentric square that covered $1 / 4$ of the floor area. Three in- dexes-total running distance, entries into the central area, and average speed-were used to reflect the motor function of the mice.

Compliance and ethics The author(s) declared that they have no conflict of interests. And all experiments were performed in accordance with the Helsinki Declaration of 1975 (as revised in 2008) concerning Human and Animal Rights.

Acknowledgements We thank Dr. Yaping Yan and Fudong Shi at Tianjin Medical University for helps on EAE mouse model. This work was supported by the China National Basic Research Program (2013CB966901, 2012CBA01303), the Strategic Priority Research Program of the Chinese Academy of Sciences (XDA01040108), National Thousand Young Talents Program to Tongbiao Zhao, and the National Natural Science Foundation of China Program ((31271592, 31570995) to Tongbiao Zhao, (31400831) to Jiani Cao).

Ager, R.R., Davis, J.L., Agazaryan, A., Benavente, F., Poon, W.W., LaFerla, F.M., and Blurton-Jones, M. (2015). Human neural stem cells improve cognition and promote synaptic growth in two complementary transgenic models of Alzheimer's disease and neuronal loss. Hippocampus 25, 813-826.

Araki, R., Uda, M., Hoki, Y., Sunayama, M., Nakamura, M., Ando, S., Sugiura, M., Ideno, H., Shimada, A., Nifuji, A., and Abe, M. (2013). Negligible immunogenicity of terminally differentiated cells derived from induced pluripotent or embryonic stem cells. Nature 494, $100-104$.

Arima, Y., Harada, M., Kamimura, D., Park, J.H., Kawano, F., Yull, F.E., Kawamoto, T., Iwakura, Y., Betz, U.A.K., Ma and Abe, M. (2013). Negligible immunogenicity of terminally differendefines a gateway for autoreactive T cells to cross the blood-brain barrier. Cell 148, 447-457.

Bai, L., Hecker, J., Kerstetter, A., and Miller, R.H. (2013). Myelin repair and functional recovery mediated by neural cell transplantation in a mouse model of multiple sclerosis. Neurosci Bull 29, 239-250.

Beare, J., E., Morehouse, J., R., DeVries, W., H., Enzmann, G., U., Burke, D., A., Magnuson, D., S.K., and Whittemore, S., R. (2009). Gait analysis in normal and spinal cotused mice using the treadscan system. J Neurotrauma 11, 2045-2056.

Ben-Hur, T., Idelson, M., Khaner, H., Pera, M., Reinhartz, E., Itzik, A., and Reubinoff, B.E. (2004). Transplantation of human embryonic stem cell-derived neural progenitors improves behavioral deficit in Parkinsonian rats. Stem Cells 22, 1246-1255.

Cao, J., Li, X., Lu, X., Zhang, C., Yu, H., and Zhao, T. (2014). Cells derived from iPSC can be immunogenic-yes or no? Protein Cell 5, 1-3.

Compston, A., and Coles, A. (2008). Multiple sclerosis. Lancet 372, 1502-1517.

Constantinescu, C.S., Farooqi, N., O’Brien, K., and Gran, B. (2011). Experimental autoimmune encephalomyelitis (EAE) as a model for multiple sclerosis (MS). Br J Pharmacol 164, 1079-1106.

Crusio, W., E. (2001). Genetic dissection of mouse exploratory behaviour. Behav Brain Res 125, 127-132.

Cundiff, P.E., and Anderson, S.A. (2011). Impact of induced pluripotent stem cells on the study of central nervous system disease. Curr Opin Genet Dev 21, 354-361.

de Almeida, P.E., Meyer, E.H., Kooreman, N.G., Diecke, S., Dey, D., Sanchez-Freire, V., Hu, S., Ebert, A., Odegaard, J., Mordwinkin, N.M., Brouwer, T.P., Lo, D., Montoro, D.T., Longaker, M.T., Negrin, R.S., and Wu, J.C. (2014). Transplanted terminally differentiated induced pluripotent stem cells are accepted by immune mechanisms similar to self-tolerance. Nat Commun 5, 3903.

Durnaoglu, S., Genc, S., and Genc, K. (2011). Patient-specific pluripotent stem cells in neurological diseases. Stem Cells Int 2011, 212487.

Einstein, O., Karussis, D., Grigoriadis, N., Mizrachi-Kol, R., Reinhartz, E., Abramsky, O., and Ben-Hur, T. (2003). Intraventricular transplantation 
of neural precursor cell spheres attenuates acute experimental allergic encephalomyelitis. Mol Cell Neurosci 24, 1074-1082.

Fassas, A., Anagnostopoulos, A., and Tsompanakou1, A. (1997). Peripheral blood stem cell transplantation in the treatment of progressive multiple sclerosis: first results of a pilot study. Bone Marrow Transplant 20, 631-638.

Fisher-Shoval, Y., Barhum, Y., Sadan, O., Yust-Katz, S., Ben-Zur, T., Lev, N., Benkler, C., Hod, M., Melamed, E., and Offen, D. (2012). Transplantation of placenta-derived mesenchymal stem cells in the EAE mouse model of MS. J Mol Neurosci 48, 176-184.

Harris, V.K., Yan, Q.J., Vyshkina, T., Sahabi, S., Liu, X., and Sadiq, S.A. (2012). Clinical and pathological effects of intrathecal injection of mesenchymal stem cell-derived neural progenitors in an experimental model of multiple sclerosis. J Neurol Sci 313, 167-177.

Huang, S., and Fu, X. (2014). Stem cell therapies and regenerative medicine in China. Sci China Life Sci 57, 157-161.

Israel, M.A., Yuan, S.H., Bardy, C., Reyna, S.M., Mu, Y., Herrera, C., Hefferan, M.P., Van Gorp, S., Nazor, K.L., Boscolo, F.S., Carson, C.T., Laurent, L.C., Marsala, M., Gage, F.H., Remes, A.M., Koo, E.H., and Goldstein, L.S. (2012). Probing sporadic and familial Alzheimer's disease using induced pluripotent stem cells. Nature 482, 216-220.

Jelinek, G.A., Weiland, T.J., Hadgkiss, E.J., Marck, C.H., Pereira, N., and van der Meer, D.M. (2015). Medication use in a large international sample of people with multiple sclerosis: associations with quality of life, relapse rate and disability. Neurol Res 37, 662-673.

Kim, H., Walczak, P., Kerr, C., Galpoththawela, C., Gilad, A.A., Muja, N., and Bulte, J.W. (2012). Immunomodulation by transplanted human embryonic stem cell-derived oligodendroglial progenitors in experimental autoimmune encephalomyelitis. Stem Cells 30, 2820-2829.

Kim, J.H., Auerbach, J.M., Rodriguez-Gomez, J.A., Velasco, I., Gavin, D., Lumelsky, N., Lee, S.H., Nguyen, J., Sanchez-Pernaute, R., Bankiewicz, K., and McKay, R. (2002). Dopamine neurons derived from embryonic stem cells function in an animal model of Parkinson's disease. Nature 418, 50-56.

Kleinschmidt-DeMasters, B.K., and Tyler K, L. (2005). Progressive multifocal leukoencephalopathy complicating treatment with natalizumab and interferon beta-1a for multiple sclerosis. N Engl J Med 353, 369-374.

Lajimi, A.A., Hagh, M.F., Saki, N., Mortaz, E., Soleimani, M., and Rahim, F. (2012). Feasibility of cell therapy in multiple sclerosis: a systematic review of 83 studies. Int J Hematol Oncol Stem Cell Res 7, 15-33.

Lee, S.T., Chu, K., Park, J.E., Lee, K., Kang, L., Kim, S.U., and Kim, M. (2005). Intravenous administration of human neural stem cells induces functional recovery in Huntington's disease rat model. Neurosci Res $52,243-249$.
Liu, K., Song, Y., Yu, H., and Zhao, T. (2014). Understanding the roadmaps to induced pluripotency. Cell Death Dis 5, e1232.

Lu, X., and Zhao, T. (2013). Clinical therapy using iPSCs: hopes and challenges. Genomics Proteomics Bioinformatics 11, 294-298.

Okabe, S., Nilsson, K., F., Spiro, A., C., Segal, M., and McKay, R., D.G (1996). Development of neuronal precursor cells and functional postmitotic neurons from embryonic stem cells in vitro. Mech Dev 59, 89-102.

Pluchino, S., Gritti, A., Blezer, E., Amadio, S., Brambilla, E., Borsellino, G., Cossetti, C., Del Carro, U., Comi, G., t Hart, B., Vescovi, A., and Martino, G. (2009). Human neural stem cells ameliorate autoimmune encephalomyelitis in non-human primates. Ann Neurol 66, 343-354.

Pluchino, S., and Martino, G. (2008). The therapeutic plasticity of neural stem/precursor cells in multiple sclerosis. J Neurol Sci 265, 105-110.

Pluchino, S., Quattrini, A., Brambilla, E., Gritti, A., Salani, G., Dina, G., Galli, R., Del Carro, U., Amadio, S., and Bergami, A. (2003). Injection of adult neurospheres induces recovery in a chronic model of multiple sclerosis. Nature 422, 688-694.

Redmond, D.E., Jr., Bjugstad, K.B., Teng, Y.D., Ourednik, V., Ourednik, J., Wakeman, D.R., Parsons, X.H., Gonzalez, R., Blanchard, B.C., Kim, S.U., Gu, Z., Lipton, S.A., Markakis, E.A., Roth, R.H., Elsworth, J.D., Sladek, J.R., Jr., Sidman, R.L., and Snyder, E.Y. (2007). Behavioral improvement in a primate Parkinson's model is associated with multiple homeostatic effects of human neural stem cells. Proc Natl Acad Sci USA 104, 12175-12180.

Takahashi, K., and Yamanaka, S. (2006). Induction of pluripotent stem cells from mouse embryonic and adult fibroblast cultures by defined factors. Cell 126, 663-676.

Viglietta, V., Baecher-Allan, C., Weiner, H.L., and Hafler, D.A. (2004). Loss of functional suppression by $\mathrm{CD} 4{ }^{+} \mathrm{CD} 25^{+}$regulatory $\mathrm{T}$ cells in patients with multiple sclerosis. J Exp Med 199, 971-979.

Wu, S., Li, K., Yan, Y., Gran, B., Han, Y., Zhou, F., Guan, Y.T., Rostami, A., and Zhang, G.X. (2013). Intranasal delivery of neural stem cells: a CNS-specific, non-invasive cell-based therapy for experimental autoimmune encephalomyelitis. J Clin Cell Immunol 4, doi: 10.4172/21559899.1000142.

Ying, Q.L., Stavridis, M., Griffiths, D., Li, M., and Smith, A. (2003). Conversion of embryonic stem cells into neuroectodermal precursors in adherent monoculture. Nat Biotechnol 21, 183-186.

Zhao, T., Zhang, Z.N., Rong, Z., and Xu, Y. (2011). Immunogenicity of induced pluripotent stem cells. Nature 474, 212-215.

Zhao, T., Zhang, Z.N., Westenskow, P.D., Todorova, D., Hu, Z., Lin, T., Rong, Z., Kim, J., He, J., Wang, M., Clegg, D.O., Yang, Y.G., Zhang, K., Friedlander, M., and Xu, Y. (2015). Humanized mice reveal differential immunogenicity of cells derived from autologous induced pluripotent stem cells. Cell Stem Cell 17, 353-359.

Open Access This article is distributed under the terms of the Creative Commons Attribution License which permits any use, distribution, and reproduction in any medium, provided the original author(s) and source are credited. 